\title{
Experimental and Simulation Investigation of Micro- and Nano-Structured Neutron Detectors
}

\author{
Faruk Logoglu ${ }^{1}$, Patrick Albert ${ }^{2,3}$, Douglas Wolfe ${ }^{2,3}$, and Marek Flaska ${ }^{1}$ \\ 1) Ken and Mary Alice Lindquist Department of Nuclear Engineering \\ 2) Department of Materials Science and Engineering \\ 3) Applied Research Laboratory \\ The Pennsylvania State University \\ Corresponding author: ful51@ @su.edu ${ }^{1}$
}

\begin{abstract}
We are investigating different micro- and nanostructure approaches to neutron detection based on inorganic scintillators. Specifically, we have been assessing various neutron converter-scintillator configurations through simulations and experiments. One promising inorganic scintillator is $\mathrm{ZnO}$ due to its relatively high light yield ${ }^{[1]}$, reasonable optical transparency in the visible region $^{[2]}$, and relatively low refractive index ${ }^{[3]}$ compared to other $\mathbf{Z n}$-based crystals such as $\mathbf{Z n S}^{[4]}$. Accurate optical data and rigid simulation tools are necessary to optimize the dimensions of the neutron converter/scintillator systems. Accurate optical data are necessary since the optical parameters of a material depend on a variety of factors, including but not limited to its morphology, crystal structure, surface quality (surface roughness), as well as the temperature at which it was manufactured. Therefore, literature data show significant discrepancy when it comes to the optical parameters for the material and it is important to accurately measure these quantities for the specific sample of interest. Neutron detection is a complex process that includes neutron transport, charged particle transport, and light transport in the active detection medium. Hence, a rigid simulation tool is required to handle all these different areas of physics with sufficient accuracy. In this work, Geant4 has been chosen to carry out the simulations of these processes. Geant4 (GEometry ANd Tracking) is a toolkit used in various applications including high energy physics, astrophysics, and radiation detection ${ }^{[5]}$. The optical simulation capabilities of Geant 4 have been validated by comparing the transmission and reflection data from $\mathrm{UV}-\mathrm{V}$ is spectroscopy to the Geant4 models for different $\mathrm{Zn}$-based crystals. After validating the optical response of single crystals, simulation models were constructed to model more complex structures of $\mathrm{ZnS}$-based alpha detection sheets (EJ440) from Eljen Technology. Optical parameters validated with experimental results have been used in radiation simulation in Geant4. This study will serve as a basis for our ongoing effort to optimize and manufacture an efficient and compact fast neutron detection module with micro- and nano-structures.
\end{abstract}

Keywords - Spectroscopy, Geant4, Optimization, Neutron Detectors, Scintillation

\section{INTRODUCTION}

$\mathrm{F}$ AST neutron detection is achieved through either neutron scattering or neutron capture reactions. Scattering-based fast neutron detection systems typically employ hydrogen atoms due to the similar mass to that of neutrons. On the other hand, capture-based neutron detection systems utilize isotopes that have high neutron capture cross section. Three of the most important neutron capture reactions for neutron detection are given here ${ }^{[6]}$ :

$$
\begin{gathered}
{ }^{3} \mathrm{He}+n \rightarrow{ }^{3} \mathrm{H}+{ }^{1} \mathrm{H}+0.764 \mathrm{MeV} \\
{ }^{6} \mathrm{Bi}+n \rightarrow n \rightarrow\left\{\begin{array}{c}
{ }^{7} \mathrm{He}+{ }^{3} \mathrm{H}+4.8 \mathrm{MeV} \\
\mathrm{Li}+{ }^{4} \mathrm{He}+2.31 \mathrm{MeV}+0.48 \mathrm{MeV}(\gamma)(94 \%) \\
{ }^{7} \mathrm{Li}+{ }^{4} \mathrm{He}+2.79 \mathrm{MeV}(6 \%)
\end{array}\right\}
\end{gathered}
$$

Although ${ }^{3} \mathrm{He}$ has superior thermal neutron capture cross section (5333 barns) than those of ${ }^{6} \mathrm{Li}$ (940 barns) and ${ }^{10} \mathrm{~B}(3842$ barns $)^{[6]}$, its worldwide shortage prohibits its widespread use in newly developed detectors ${ }^{[7]}$. This aforementioned shortage of ${ }^{3} \mathrm{He}$ has led researchers to develop neutron detectors based on alternative approaches ${ }^{[8]}$.

In our recent studies, we have investigated several approaches to maximize the neutron detection efficiency of various neutron converter/scintillator configurations ${ }^{[9]}$. Optimizations of different components of proposed neutron detectors were carried out in Geant4 simulations. Although neutronic data and charged particle models are relatively consistent between Geant 4 , MCNP6 ${ }^{[10]}$, and experiments ${ }^{[7]}$, optical data have to be accurately measured for the material samples of interest. The difficulty arises due to the fact that optical constants are not only dependent on elemental composition of the materials, but also on their morphology, crystal structure, surface roughness, and the temperature at which they were manufactured. Therefore, optical parameters of the specific detector components should be measured, and those same optical parameters must be used in optimization and validation studies.

Moreover, the simulation tool has to be capable of accurately capturing different optical phenomena. These optical phenomena include refraction, surface reflection, and total internal reflection, as well as bulk refraction and bulk reflection 
for samples with a polymeric-matrix containing particles, such as EJ-440 detection sheets from Eljen Technologies.

In this work, we are investigating how Geant4 models compare against the experimental results of transmission/reflection spectroscopy for samples with thicknesses larger than/comparable to the wavelength of electromagnetic radiation in the UV-Vis region.

\section{EXPERIMENTS}

A $\mathrm{ZnO}$ sample (99.99\% purity) with dimensions of $10 \mathrm{~mm} \mathrm{x}$ $10 \mathrm{~mm} \times 0.5 \mathrm{~mm}$ was obtained from MSE supplies (Tucson, AZ). A ZnS sample with 1-inch diameter and $3.175 \mathrm{~mm}$ thickness was obtained from II-VI Incorporated (Saxonburg, PA). Two alpha detection sheets (EJ-440) were obtained from Eljen Technologies (Sweetwater, TX) with two different thicknesses of $0.1 \mathrm{~mm}$ and $0.25 \mathrm{~mm}$. The EJ-440 sheets consist of $\mathrm{ZnS}(\mathrm{Ag})$ powders bound to a $0.25-\mathrm{mm}$ thick polyester sheets on only one side, which allows Scanning Electron Microscopy (SEM) images to be taken off the surface of the samples. Moreover, several polyester sheets with $0.25-\mathrm{mm}$ thickness were obtained from Eljen Technologies to make separate transmission/reflection measurement to decouple the effect of polyester sheets from the EJ-440 responses.

Micro- and nano-structured $\mathrm{ZnO}$ and boron samples with different thicknesses were manufactured in Material Research Institute (MRI) at Pennsylvania State University (PSU). Wavelength dependent refractive indices and extinction coefficients of these samples were measured with Woollam M2000F Focused Beam Spectroscopic Ellipsometer. Transmission/reflection spectra were measured with Cary-5000 UV-Vis-NIR spectrophotometer with an integrating sphere attachment.

SEM images of EJ-440 samples were taken to calculate the average particle dimensions of $\mathrm{ZnS}(\mathrm{Ag})$ powders, as well as the standard deviation of their sizes. Iridium coating was performed on the samples before SEM images were taken in order to eliminate the charging and to make the images clearer. It was observed through several different SEM images that the samples were relatively homogenous in powder distribution. Line-intercept analysis was conducted to find the mean particle size and the standard deviation using SEM images. Additionally, Energy Dispersive Spectroscopy (EDS) was conducted to observe the impurities in the samples.

\section{DATA ANALYSIS}

UV-VIS spectroscopy results for transmission and reflection were analyzed, and the optical absorption coefficients of the samples were calculated. Measured transmission and reflection can be related to the bulk absorption coefficient and the surface reflection coefficient as follows for a single layer optical system $^{[11]}$ :

$$
\begin{gathered}
T_{m}=\frac{(1-R)^{2} e^{-\alpha d}}{1-R^{2} e^{-2 \alpha d}} \\
R_{m}=R+\frac{R(1-R)^{2} e^{-2 \alpha d}}{1-R^{2} e^{-2 \alpha d}}
\end{gathered}
$$

where $\mathrm{T}_{\mathrm{m}}, \mathrm{R}_{\mathrm{m}}, \alpha, \mathrm{R}$, and $\mathrm{d}$ are the measured transmission, measured reflection, bulk absorption coefficient, surface reflection coefficient, and sample thickness, respectively. Reflection coefficient can be related to the refractive index of the material for normal incidence for samples with insignificant absorption through ${ }^{[11]}$ :

$$
R=\frac{(n-1)^{2}}{(n+1)^{2}}
$$

where $n$ is the refractive index of the crystal. Equations 2 and 3 can be solved numerically to find the bulk absorption coefficient and the surface reflection coefficient simultaneously.

In most of the literature, the absorption coefficient is calculated with the Beer-Lambert Law and the so-called "Modified Beer-Lambert Law"[11]. Although these models can be applied to many samples, it is important to understand their limitations. The Beer-Lambert Law and the Modified BeerLambert Law are given as follows:

$$
\begin{gathered}
T_{m}=e^{-\alpha d} \\
T_{m}=\left(1-R_{m}\right)^{2} e^{-\alpha d}
\end{gathered}
$$

Both of these equations give accurate results in certain regions of the transmission/reflection spectrum; however, they do not give plausible results when their approximations are no longer valid. As an example, Equation 5 is only valid when the measured reflection is negligible compared to the measured transmission in Equations 2 and 3. On the other hand, Equation 6 is only valid when the sample is considerably absorbent ${ }^{[11]}$. These limitations will be shown through Geant 4 simulations in Results and Discussion.

For two-layer systems such as those in EJ-440 sheets, none of the equations discussed so far gives accurate results due to the additional polyester backing. Polyester backing introduces an extra bulk region, as well as two surfaces with different refractive index mismatch (ZnS-polyester and polyester-air). Therefore, Transfer Matrix Method (TMM) ${ }^{[12]}$ was used to extract the absorption coefficient of the active detection region for these samples. Expressions for the transmission and reflection of a two-layer system are fairly complicated; as an example, measured transmission of a ZnS-Polyester system when the light is impinged from $\mathrm{ZnS}$ side is expressed as follows:

$$
T_{Z n S}=\frac{\left(1-R_{1}\right)\left(1-R_{2}\right)\left(1-R_{3}\right)}{\left\{\left[\left(1-2 R_{2}\right) R_{3} e_{2}^{-1} e_{1}^{-1}+R_{2} e_{2} e_{1}^{-1}\right]\left(-R_{1}\right)-R_{2} R_{3} e_{2}^{-1} e_{1}+e_{2} e_{1}\right\}}
$$

where $R_{1}, R_{2}, R_{3}$ are the reflection coefficients of air- $\mathrm{ZnS}, \mathrm{ZnS}$ polyester, and polyester-air surfaces, respectively. $e_{1}$ and $e_{2}$ are given by:

$$
e_{1}=e^{\alpha_{1} d_{1}}, \quad e_{2}=e^{\alpha_{2} d_{2}}
$$

where $\alpha_{1}, \alpha_{2}, \mathrm{~d}_{1}$, and $\mathrm{d}_{2}$ are the $\mathrm{ZnS}$ absorption coefficient, polyester absorption coefficient, $\mathrm{ZnS}$ thickness, and polyester thickness, respectively. Similarly, there are the measured reflection when light is impinged on $\mathrm{ZnS}$ side, as well as the 
measured transmission and reflection data when light is impinged on polyester side, which, in total, amount to four measured values. If the absorption coefficient of polyester was measured separately using Equations 2 and $3, \alpha_{1}, R_{1}, R_{2}$, and $R_{3}$ can be found by solving four equations simultaneously.

Wave nature of light must be taken into account while analyzing the UV-Vis spectra of thin samples. The wave nature of light gives rise to interference fringes due to multiple reflections of light waves inside the thin sample. A mathematical expression can be used to represent these effects in order to extract the absorption coefficients of the samples. The mathematical expression of transmission for double-layer systems involving a thin film and a thick substrate is shown here $^{[13]}$ :

$$
T=\frac{\left|t_{12} t_{23} t_{34}\right|^{2}\left|e_{2}\right|^{2}\left|e_{3}\right|^{2}}{\left|1+r_{23} r_{34} e_{3}^{2}+r_{12} r_{23} e_{2}^{2}+r_{12} r_{34} e_{2}^{2} e_{3}^{2}\right|^{2}}
$$

where $t_{n m}$ and $r_{n m}$ are the transmission and reflection coefficients, respectively. The term $\mathrm{e}_{\mathrm{x}}$ can be expressed as

$$
e_{x}=e^{-2 \pi \kappa_{x} k d_{2}} e^{2 \pi i n_{x} k d_{2}}
$$

where $\kappa_{x}$ and $\mathrm{n}_{\mathrm{x}}$ are the extinction coefficient and refractive index of the material of interest, respectively, and $\mathrm{k}$ is the wavenumber.

Particle size analysis was carried out using line-intercept analysis on SEM micrographs from a Tescan FESEM. The analysis processes used multiple lines drawn both horizontally and vertically on the images. The number of particles crossed by the lines was determined and the average particle size was calculated by dividing the total line length by the number of particles. Standard deviation of particle size was calculated through error propagation using the uncertainty in the number of particles crossed by the intercepting lines.

Mean particle size and the standard deviation are found using

$$
\begin{gathered}
\mu=\frac{L}{N} \\
\sigma_{\mu}=\mu \frac{\sigma_{N}}{N}
\end{gathered}
$$

where $\mu, \mathrm{L}$, and $\mathrm{N}$ are the mean particle size, total line length, and total number of particles intercepted, respectively. $\sigma_{\mu}$ and $\sigma_{N}$ are the uncertainties in mean particle size and number of particles intercepted, respectively.

\section{Computational Methods}

For the simulations of $\mathrm{ZnS}$ and $\mathrm{ZnO}$ crystals, a single volume was created in Geant 4 and a circular polychromatic beam of light was impinged on one side of the target to mimic the experimental setup. No surface definition was used in the simulations; therefore, probabilities of reflection and refraction are calculated directly from the refractive index data found in the literature, which were verified with the experiments using Equation 4. Transmission and reflection are determined by tallying the total number of photons that escaped the crystal through the back surface and the front surface (surface on which light is impinged), respectively, and dividing them by the total number of photons simulated. Similar simulation setup was utilized for thin films with only difference being the addition of quartz substrate into the models. Simulations were conducted using the optical constants measured with ellipsometry and verified with UV-Vis spectroscopy. Moreover, the neutron detection efficiency of a single sandwich layer ( $\mathrm{ZnO}$-boron$\mathrm{ZnO})$ was calculated with Geant 4 simulations.

The EJ-440 sheets were simulated by modeling the individual powders, size of which was measured with SEM images. Modeling of individual powders was found necessary to accurately reflect the complex nature of the detection sheets. When EJ-440 was modeled as a single layer, simulations severely underestimated the amount of reflected light due to no reflection inside the sample. However, when powders were modeled, reflection results in Geant4 converged to the experimental data due to the multiple reflections taken place inside the sample.

A Gaussian function was written in Geant4 DetectorConstruction class with the mean particle size and standard deviation information taken from SEM images. For EJ-440, spherical particles ( $\mathrm{ZnS}(\mathrm{Ag})$ powders) with pseudorandomized radii were placed in a cubical volume of air. Positions of the particles in the cubical volume were also randomized. The cubical volume containing the $\mathrm{ZnS}(\mathrm{Ag})$ powders were repeated and the entire active volume of the detectors was filled with spherical $\mathrm{ZnS}(\mathrm{Ag})$. Finally, a $0.25 \mathrm{~mm}$ polyester sheet was created and placed behind the $\mathrm{ZnS}(\mathrm{Ag})$ volume.

\section{RESULTS AND DISCUSSION}

We investigated the optical transport capabilities of Geant4 toolkit for single crystal samples, complex powder-based samples, and thin samples. We used measured data from ellipsometry, transmission/reflection spectroscopy and SEM in Geant 4 simulations. Results for different samples are shown in the following subsections.

\section{A. ZnS Crystal}

Bulk absorption coefficient of the $\mathrm{ZnS}$ crystal was calculated using the three methods discussed in Section III and wavelength dependent absorption length $(1 / \alpha)$ was entered as input into the Geant4 model. Refractive index data were taken from literature ${ }^{[14]}$. The wavelength dependent refractive index was also calculated through Equations 2-4 and compared to the literature values. Experimental and simulation results for transmission/reflection/absorption spectra are shown in Fig. 1.

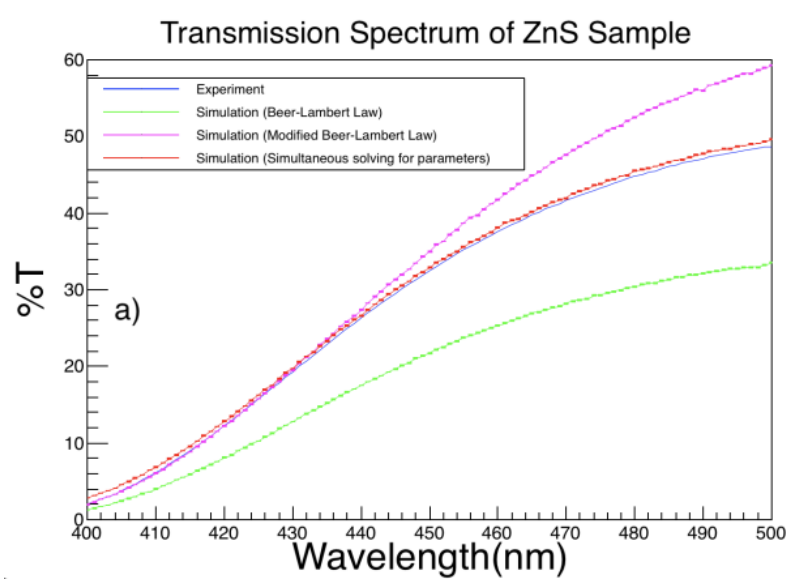



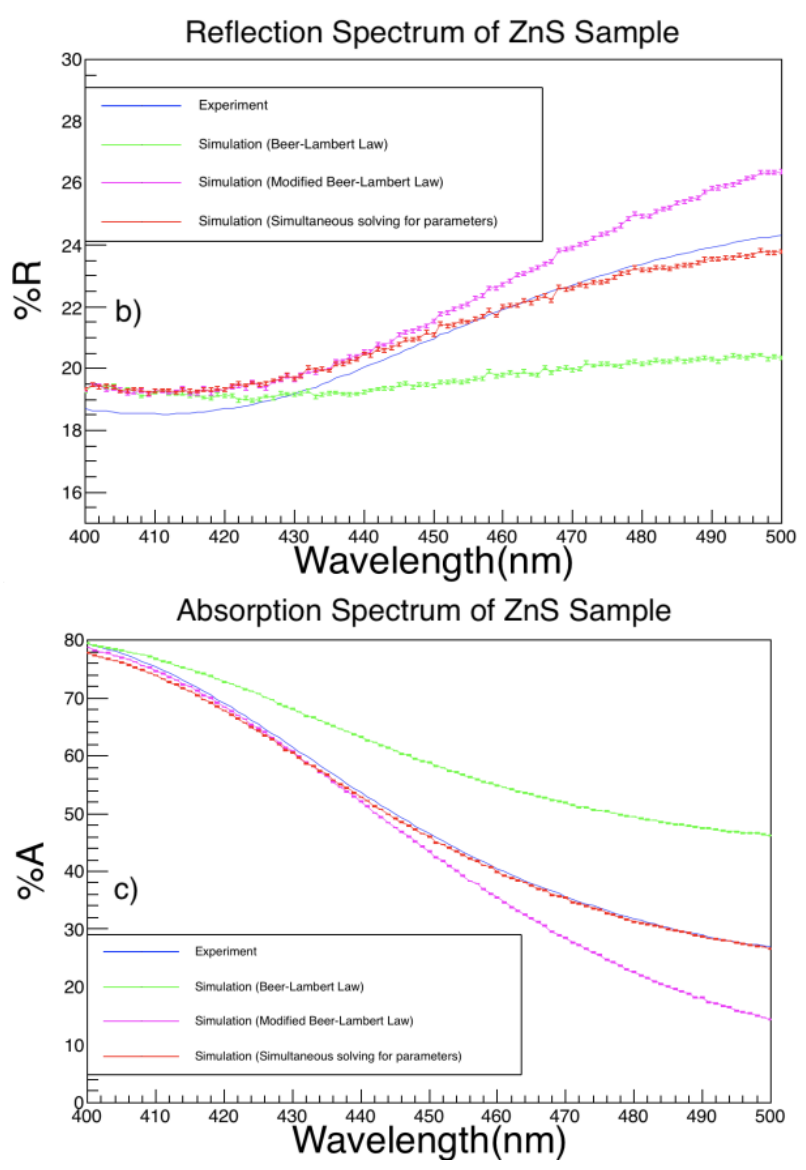

Figure 1. a) Transmission, b) reflection, and c) aAbsorption spectra of the $\mathrm{ZnS}$ sample. Both experimental and simulation data are shown.

As shown in Fig.1, transmission increases as the wavelength of the light photon increases (or energy of the photon decreases). Experimental data are consistent with the data sheet provided by the company. As stated in Section III of this paper, both the Beer-Lambert Law and the Modified Beer-Lambert Law capture certain parts of the spectra accurately. However, simultaneous calculation of bulk absorption and reflection coefficients through Equations 2 and 3 give better results in the whole wavelength region. The Beer-Lambert Law fails significantly as reflection increases, whereas the Modified Beer-Lambert Law fails where absorbance is minimal. Since the Beer-Lambert Law completely ignores reflection, it overestimates the absorption in the entirety of the spectrum.

Uncertainties in the simulations are less than $0.2 \%$ and they are shown in the figures. Uncertainty estimation in UV-Vis analysis was reported to be $0.15 \%-1 \%{ }^{[15,16]}$.

The largest percentage difference between the experimental and simulation results was found in the reflection spectrum with the largest difference being $4.5 \%$. The reason for this difference was attributed to the inaccurate refractive index data and imprecise surface definitions used in Geant 4 models that ignored possible surface roughness. Refractive indices calculated with Equation 4 were on average 5\% lower than the literature values. When the simulations were done with the calculated refractive indices, we found that the reflection decreased and matched with the experiments in higher photon energy region; however, it deviated from the experiments in the lower photon energy region.

\section{B. ZnO Crystal}

The wavelength dependent bulk absorption coefficient of $\mathrm{ZnO}$ crystal was calculated through Equations 2 and 3. The sample cross-sectional area was $10 \mathrm{~mm}$ x $10 \mathrm{~mm}$; therefore, a micro-focusing lens was used to collimate the light onto the center of the sample. Refractive indices calculated with Equation 4 were used in the Geant 4 models. Spectra calculated from the simulations were compared against the experimental spectra. As an example, the absorption spectrum is shown in Fig. 2.

We observed a very good match between the experimental and simulation results in the absorption spectrum. The highest difference between the experiments and simulations was around $2 \%$ in the low energy region of the spectrum. Although not shown here, we observed the largest difference between experiments and simulations in the reflection spectrum with the highest relative error being approximately $9.5 \%$. We assign this discrepancy to the combination of imprecise data of refractive index and non-realistic surface conditions in the models.

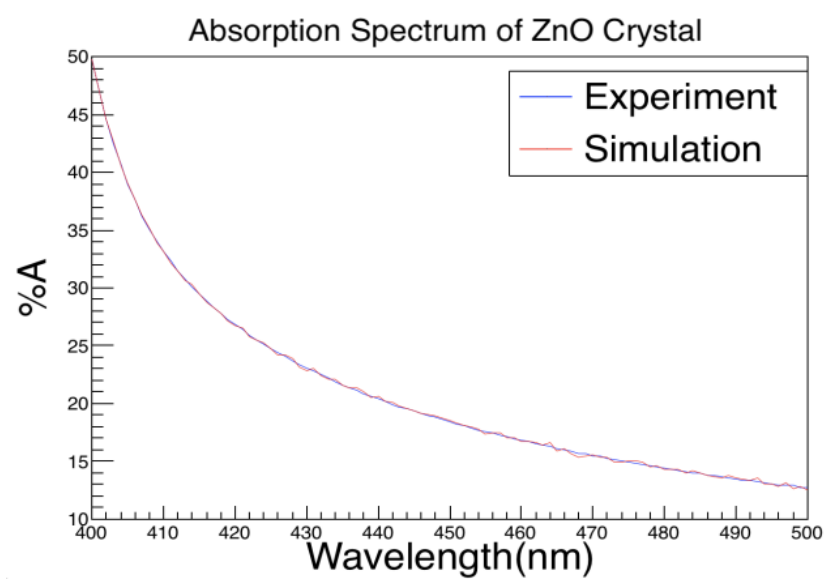

Figure 2. Comparison of experimental and simulation absorption spectra.

\section{EJ-440 Sheets}

Transmission/reflection spectroscopy measurements were carried out for EJ-440 sheets with two different thicknesses. Since these sheets are made up of two layers $(\mathrm{ZnS}(\mathrm{Ag})$ and polyester), a total of four measurements were taken for each sample with two transmission and two reflection measurements with light impinged on both sides of the samples.

Moreover, UV-Vis measurements were carried out for a single polyester layer obtained from Eljen Technologies to find the bulk absorption coefficient of the polyester backing. The polyester bulk absorption coefficient ( $\alpha_{2}$ in Equation 8 ) was used to determine the bulk absorption coefficient of $\mathrm{ZnS}(\mathrm{Ag})$ layer using Equation 7 and the additional three equations discussed in Section III (not explicitly shown in Section III).

The mean particle size was calculated as $4.4 \mu \mathrm{m}$ and $4.5 \mu \mathrm{m}$ for $0.1 \mathrm{~mm}$ and $0.25 \mathrm{~mm}$ EJ-440 sheets, respectively. The standard deviations associated to those values were calculated to be $0.88 \mu \mathrm{m}$ and $0.76 \mu \mathrm{m}$. The active detector volume was created in Geant 4 with spherical $\mathrm{ZnS}(\mathrm{Ag})$ powders whose sizes 
were pseudorandomized through the following probability density function (PDF) with experimentally verified mean and standard deviation:

$$
f(x)=\frac{1}{\sqrt{2 \pi \sigma}} e^{\frac{-(x-\mu)^{2}}{2 \sigma^{2}}}
$$

where $\mu$ and $\sigma$ are the mean particle size and standard deviation associated to it, respectively.

An SEM image taken with the secondary electron detector is shown in Fig. 3 for a $0.1-\mathrm{mm}$ thick EJ-440 sheet. Highly complex internal structure of the powdery geometry of EJ-440 sheets was approximated by using spheres with pseudorandomized radii calculated with Equation 13. Although the model falls short to mimic the true structure, it nonetheless introduces complicated passage for light and therefore causes light to reflect off multiple boundaries inside the bulk material. A unit-cell created in Geant4 using the data obtained from SEM images is shown in Fig. 4.

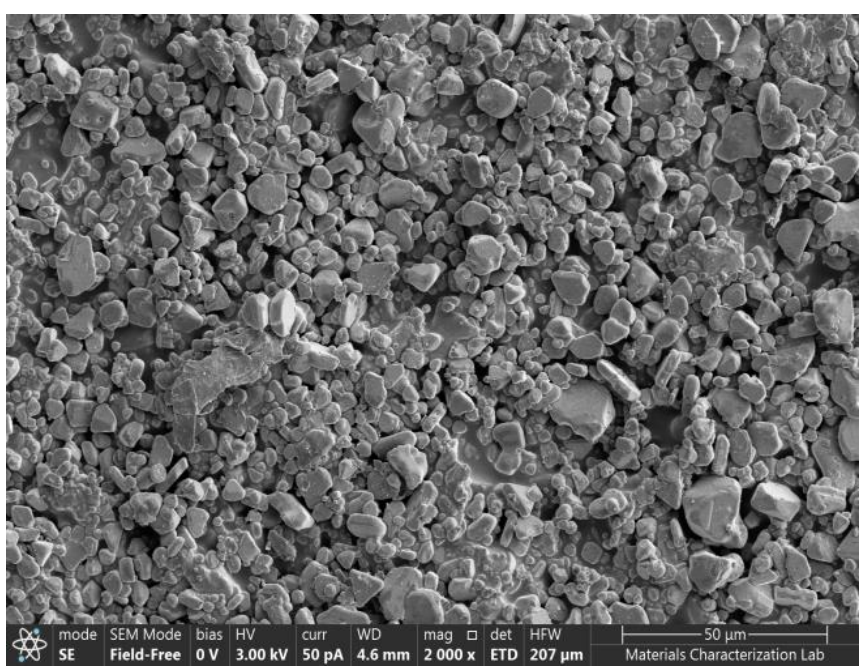

Figure 3. SEM image for 0.1-mm thick EJ-440 sheet.

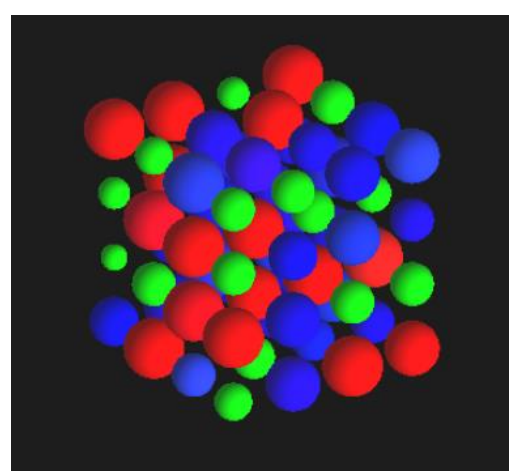

Figure 4. Unit-cell in Geant4 for EJ-440 sheets. Particles are color coded according to their sizes. All spheres are ZnS(Ag) with green representing smaller than $\mu-\sigma$, blue representing between $\mu-\sigma$ and $\mu+\sigma$, and red representing larger than $\mu+\sigma$.

All the volumes shown in Fig. 4 are $\mathrm{ZnS}(\mathrm{Ag})$ with red representing particles larger than $\mu+\sigma$, blue representing particles whose sizes vary between $\mu-\sigma$ and $\mu+\sigma$, and green representing particles smaller than $\mu-\sigma$. The unit-cell was copied and repeated to create the active volume that represents the EJ-440 sheet. A polyester sheet was defined as a single rectangular-parallelepiped volume and placed behind the EJ440 active volume.

Absorption spectra obtained from the simulations for $0.1-\mathrm{mm}$ and $0.25-\mathrm{mm}$ thick EJ-440 samples were compared to the experimental spectra. Moreover, the spectra simulated using the absorption length obtained by Beer-Lambert Law were also compared to the experimental spectra. The comparison is shown in Fig. 5.

The Beer-Lambert Law severely overestimates the absorption in the sample in both cases. As explained in the discussion in section ZnS Crystal, the Beer-Lambert Law does not take into account the reflection and assumes that reflection is zero for all wavelengths. Consequently, all the light that is not transmitted is considered absorbed according to Equation 5; hence, the absorption is overestimated.
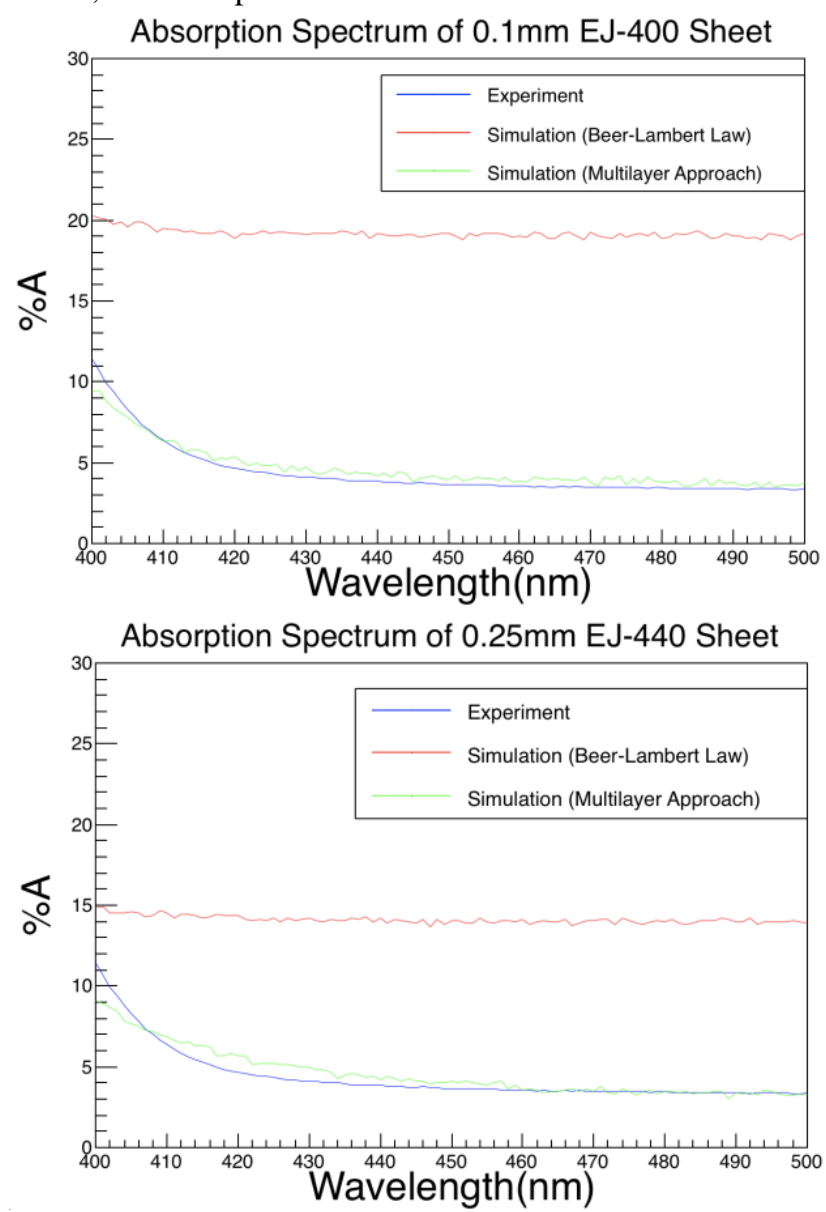

Figure 5. Comparison of experimental and simulation absorption spectra.

On the other hand, simulations with the bulk absorption coefficient calculated through the "Multilayer Approach" gives much better results and they are in agreement with the experiments in terms of both absolute values and the general trend.

Ignoring the internal structure of the samples results in a significantly lower simulated reflection. This is a result of no reflection inside the bulk material and reflection only from the surfaces between air and the sample. Introducing the $\mathrm{ZnS}(\mathrm{Ag})$ powders as micro-structures inside the bulk material causes 
incoming photons to reflect and change their paths multiple times before they leave the sample; hence, increasing the simulated reflection.

Capturing the general trend and the absolute values of absorption is only possible by taking into account the polyester layer and by modeling the internal structure of the sheets.

\section{Thin Films of $\mathrm{ZnO}$ and Boron}

Two ZnO samples with 450-nm and 939-nm thicknesses and three boron samples with 150-nm, 998-nm, and 2027-nm thicknesses were manufactured in MRI at PSU. The wavelength dependent refractive indices and extinction coefficients of these samples were measured with ellipsometry. Measured extinction coefficients and refractive indices were plugged into the model transmission (Equation 9), and the mathematical model for transmission was compared to the experimental spectrum.

SEM images of boron and $\mathrm{ZnO}$ films alongside with their transmission spectra are shown in Fig. 6.
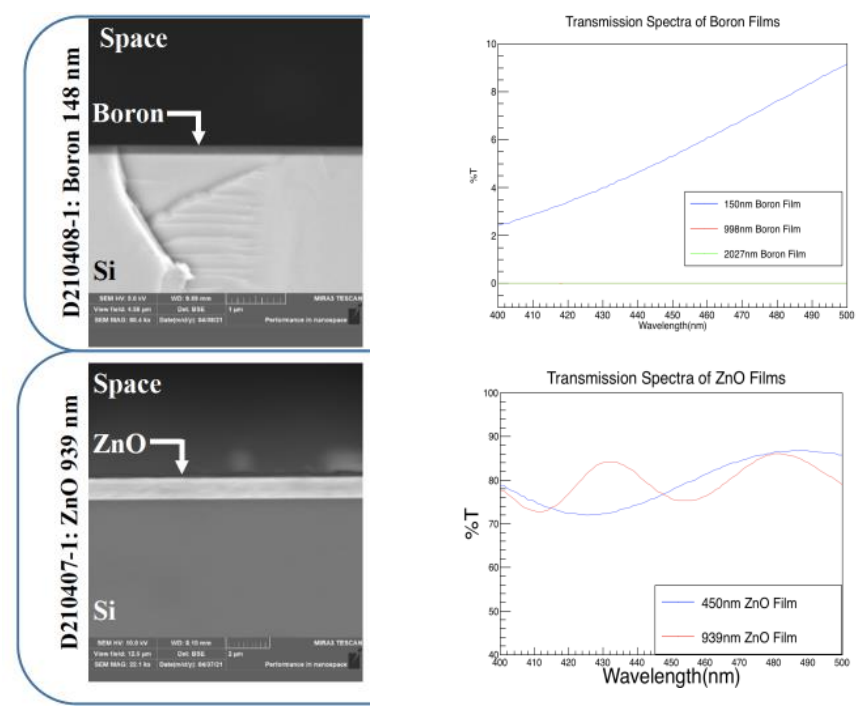

Figure 6. SEM images of $\mathrm{ZnO}$ and boron thin films with their transmission spectra.

Sample thickness and refractive indices are the main factors that determine the interference fringe pattern. There was a disagreement between the sample thicknesses measured with SEM and ellipsometry. Although the difference is less than $10 \%$ for all the samples, it changes the constructive and destructive interference pattern significantly in the model transmission spectra. These discrepancies between the ellipsometry and SEM data were taken into account while creating the model transmission. The wavelength dependent extinction coefficient and refractive index from the ellipsometry were plugged into the model to finalize the model transmission. Model transmission spectra for $450 \mathrm{~nm}$ and 939 $\mathrm{nm} \mathrm{ZnO}$ sample are shown in Fig. 7.

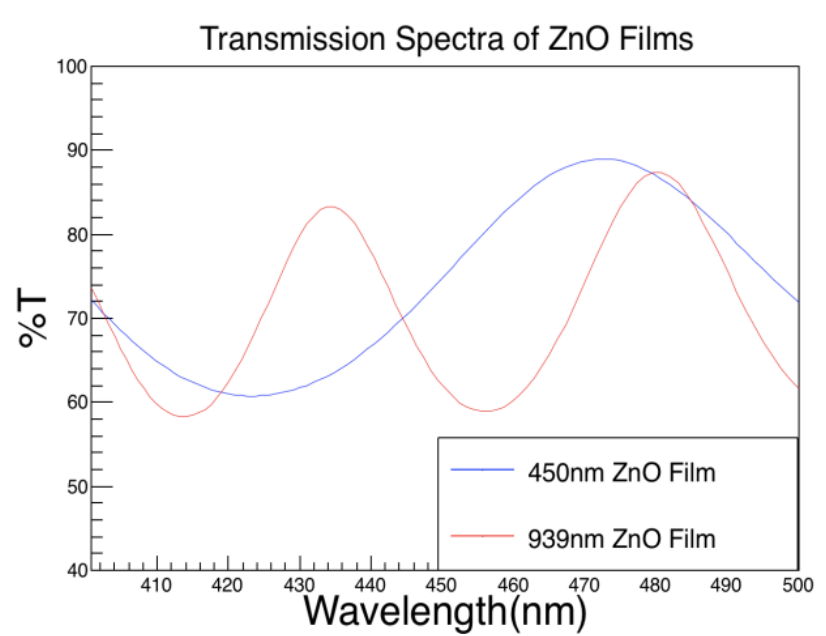

Figure 7. Model transmission for 450nm and 939nm $\mathrm{ZnO}$ thin films.

Although the experimental UV-Vis transmission and the model transmission do not match in terms of oscillation amplitudes, wavelength dependent extinction coefficient measured with ellipsometry predicts the dampening of light waves to approximately $80 \%$.

Since Geant 4 considers optical photons to be point-like particles, the interference fringes cannot be simulated in Geant4. However, Geant4 predicts a reasonable mean transmission for most of the wavelength region of interest using the ellipsometry data as shown in Fig. 8. At high energies, there is a consistent pattern of ellipsometry overestimating the extinction coefficient of the layers; therefore, Geant 4 predicts a significantly lower transmission.

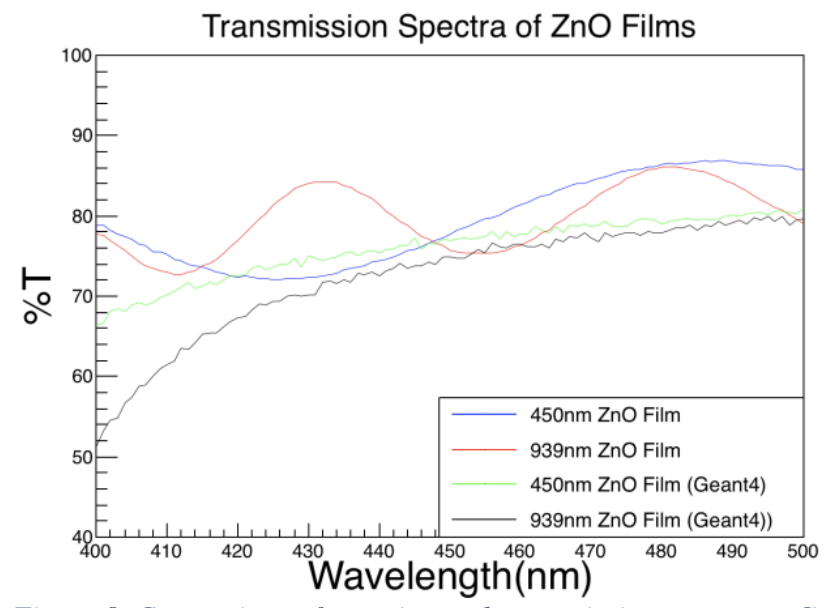

Figure 8. Comparison of experimental transmission spectra to Geant4 transmission spectra for $\mathrm{ZnO}$ thin films.

Finally, using the optical constants measured with ellipsometry and verified with UV-Vis transmission and reflection spectrometry, Geant 4 models were constructed to simulate the neutron detection efficiency of sandwich-like structures. A circular thermal neutron beam was impinged on the $\mathrm{ZnO}$-boron- $\mathrm{ZnO}$ sandwich geometry with a cross-sectional area of $3 \mathrm{~cm} \times 3 \mathrm{~cm}$. A photocathode surface was placed on one end of the geometry and the neutron capture and neutron detection efficiencies were tallied. A 20-photon threshold was applied in order to consider a successful neutron detection. The 
neutron capture efficiency and the neutron detection efficiency as a function of boron thickness are shown in Fig. 9. The $\mathrm{ZnO}$ thickness was held constant at $4 \mu \mathrm{m}$, which is the estimated range of $1.78-\mathrm{MeV}$ alpha particle in $\mathrm{ZnO}$.

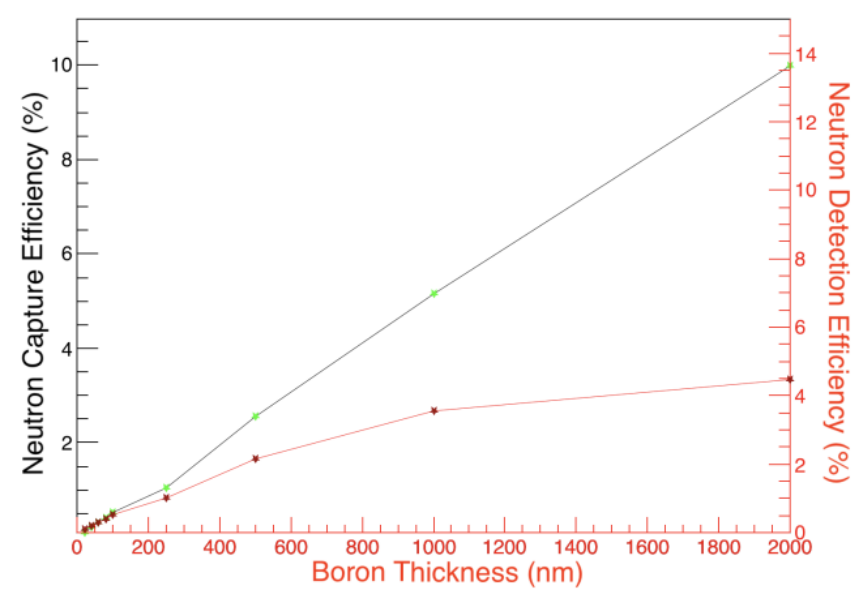

Figure 9. Neutron capture efficiency and neutron detection efficiency of $\mathrm{ZnO}-\mathrm{B}-\mathrm{ZnO}$ sandwich geometry with respect to varying boron thickness.

Although the thermal-neutron capture efficiency reaches approximately $10 \%$, the thermal-neutron detection efficiency saturates at approximately $4.5 \%$ due to the relative opaqueness of the boron film and some of the reaction products not reaching the scintillator medium. The neutron detection efficiency can be substantially increased by increasing the number of layers and by arranging multiple photosensors around the neutron detector. The neutron detection efficiency is comparable to that of similar structures for thermal neutrons ${ }^{[17]}$.

\section{CONCLUSION}

It has been shown that Geant4 is capable of modeling the optical response of various structures including single crystal and micro-structures, as well as nano-structures to a certain extent. The transmission and reflection spectra of various materials have been measured and it has been demonstrated that Geant4 accurately predicts the optical behavior of these materials when optical constants such as absorption coefficients and refractive indices are calculated with appropriate mathematical expressions. Optical constants of $\mathrm{ZnO}$ and boron thin films have been measured with ellipsometry and verified with UV-Vis spectroscopy. Finally, the neutron detection efficiency of single sandwich geometry of $\mathrm{ZnO}-\mathrm{B}-\mathrm{ZnO}$ was simulated with experimentally measured optical properties. It has been found that a single micro-structure sandwich geometry can achieve approximately $4.5 \%$ neutron detection efficiency, which is comparable to those reported previously for similar structures.

\section{ACKNOWLEDGEMENT}

This research is sponsored by the Defense Threat Reduction Agency (DTRA) as part of the Interaction of Ionizing Radiation with Matter University Research Alliance (IIRM-URA) under contract number HDTRA1-20-2-0002.

\section{REFERENCES}

[1] P. A. Rodnyi et al, "Novel Scintillation Material-ZnO Transparent Ceramics," IEEE Transactions on Nuclear Science, vol. 59, (5), pp. 2152-2155, 2012;2011;

[2] E. I. Gorokhova et al, "Optical, luminescence, and scintillation properties of $\mathrm{ZnO}$ and $\mathrm{ZnO}$ :Ga ceramics," 2010.

[3] W. L. Bond, "Measurement of the Refractive Indices of Several Crystals," Journal of Applied Physics, vol. 36, (5), pp. 1674-1677, 1965.

[4] M. Debenham, "Refractive indices of zinc sulfide in the 0405-13$\mu \mathrm{m}$ wavelength range," Applied Optics, vol. 23, (14), pp. 2238, 1984.

[5] J. Allison et al, "Recent developments in Geant4," Nuclear Instruments \& Methods in Physics Research. Section A, Accelerators, Spectrometers, Detectors and Associated Equipment, vol. 835, (C), pp. 186-225, 2016.

[6] J E McMillan et al 2015 J. Phys.: Conf. Ser. 620012011

[7] Y. Yehuda-Zada et al, "Optimization of 6LiF:ZnS(Ag) scintillator light yield using GEANT4," Nuclear Instruments \& Methods in Physics Research. Section A, Accelerators, Spectrometers, Detectors and Associated Equipment, vol. 892, pp. 59-69, 2018.

[8] G. Tsiledakis et al 2017 JINST 12 P09006

[9] F. Logoglu, M. Flaska and D. E. Wolfe, "Development of a CoatedMicro-Particle Neutron Detector Based on LiF/ZnS Scintillator," EPJ Web of Conferences, vol. 225, pp. 7009, 2020.

[10] C Guardiola et al 2011 JINST 6 T09001

[11] J. Vac. Sci. Technol. B 34, 04J105 (2016); https://doi.org/10.1116/1.4954211

[12] M. Claudia Troparevsky, Adrian S. Sabau, Andrew R. Lupini, and Zhenyu Zhang, "Transfer-matrix formalism for the calculation of optical response in multilayer systems: from coherent to incoherent interference," Opt. Express 18, 24715-24721 (2010)

[13] J. Appl. Phys. 111, $0.73109 \quad$ (2012); https://doi.org/10.1063/1.3700178

[14] S. Ozaki and S. Adachi, "Optical constants of cubic ZnS". Jpn. J. Appl. Phys. 32, 5008-5013 (1993)

[15] Rihua Mao, Liyuan Zhang and Ren-Yuan Zhu, "Optical and scintillation properties of inorganic scintillators in high energy physics," 2007 IEEE Nuclear Science Symposium Conference Record, 2007, pp.2285-2291

[16] Rev. Sci. Instrum. 88, 015105 https://doi.org/10.1063/1.4973633

[17] M. Roth, E. Mojaev, O. Khakhan, A. Fleider, E. Dul'Kin, M. Schie ber Composite boron nitride neutron detectors J. Cryst. Growth (2014) 